\title{
Study Oversight Authority
}

National Cancer Institute

\section{Source}

National Cancer Institute. Study Oversight Authority. NCI Thesaurus. Code C93450.

An organization with monitoring, regulatory, or supervisory authority over biomedical research at the local, regional, national, or international level for a particular study. 